\title{
Avaliação de lâminas cirúrgicas reutilizadas no LASIK pela microscopia eletrônica de varredura
}

\author{
Examination of blades used in LASIK by scanning electron microscopy
}

\author{
Francisco Seixas Soares ${ }^{1}$ \\ Fabio Henrique Casanova ${ }^{2}$ \\ Mauro Campos ${ }^{3}$ \\ Maria Cristina Nishiwaki-Dantas ${ }^{4}$ \\ Paulo Elias Correa Dantas 5
}

\footnotetext{
${ }^{1}$ Médico do Departamento de Oftalmologia da Santa Casa de Misericórdia de São Paulo - São Paulo (SP) Brasil.

${ }^{2}$ Médico do Departamento de Oftalmologia da Universidade Federal de São Paulo - Escola Paulista de Medicina - São Paulo (SP) - Brasil.

Professor, Livre docente do Departamento de Oftalmologia da Universidade Federal de São Paulo - Escola Paulista de Medicina - São Paulo (SP) - Brasil.

${ }^{4}$ Doutora pela Universidade Federal de São Paulo UNIFESP - São Paulo (SP) - Brasil.

Doutor em Medicina pela Universidade de São Paulo USP - São Paulo (SP) - Brasil.

Endereço para correspondência: Francisco Seixas Soares. Rua Botucatu, 822 - São Paulo (SP) CEP 04023-062 E-mail: frsoares@oftalmo.epm.br

Recebido para publicação em 18.04.2006

Última versão recebida em 30.06.2006

Aprovação em 30.06.2006

Os autores não têm interesse financeiro em nenhum produto ou medicamento citado neste estudo.

Nota Editorial: Depois de concluída a análise do artigo sob sigilo editorial e com a anuência dos Drs. Adriana dos Santos Forseto e Paulo Schor sobre a divulgação de seus nomes como revisores, agradecemos sua participação neste processo.
}

\begin{tabular}{|l|}
\hline RESUMO \\
\hline Objetivo: Avaliar e comparar, por microscopia eletrônica de varredura \\
(MEV), a superfície de lâminas cirúrgicas novas e sem uso com lâminas \\
utilizadas consecutivamente no LASIK (Laser in situ keratomileusis). \\
Métodos: Foram utilizadas 25 lâminas cirúrgicas AccuGlide ${ }^{\circledR}$ (Bausch \& \\
Lomb, EUA), divididas em 3 grupos: Grupo 1 (controle), cinco lâminas \\
novas e sem uso; Grupo 2, dez lâminas utilizadas duas vezes (cirurgia \\
bilateral simultânea); Grupo 3, dez lâminas utilizadas quatro vezes (duas \\
cirurgias bilaterais simultâneas e consecutivas). Cada lâmina foi analisada \\
em quatro pontos aleatórios, dois centrais e dois periféricos, com aumen- \\
tos de 50, 350 e 1.000 vezes. Resultados: Irregularidades e deposição de \\
material orgânico na superfície da lâmina foram observados em todas \\
lâminas reutilizadas (Grupos 2 e 3). Além disso, as lâminas do Grupo 3 \\
apresentaram mais irregularidades do que as do Grupo 2 nos pontos \\
centrais (p=0,0094) e periféricos (p=0,0098), bem como em relação ao \\
depósito de material orgânico (p=0,0204 and p=0,0909, respectivamente). \\
Não foram observados presença de material metálico e denteamento da \\
superfície cortante em nenhuma lâmina examinada. Conclusão: Não foi \\
observada diferença quanto à borda cortante entre lâminas novas e as \\
utilizadas até 4 vezes após LASIK. Por outro lado, observou-se depósito \\
de material orgânico e irregularidades da superfície de todas as lâminas \\
reutilizadas. As implicações clínicas destes achados precisam ser avaliadas \\
devido à possibilidade de estarem relacionados com complicações pós- \\
operatórias interlamelares comumente encontradas no LASIK. \\
\hline
\end{tabular}

Descritores: Ceratomileuse assistida por excimer laser in situ; Cirurgia a laser; Complicações pós-operatórias; Inflamação

\section{INTRODUÇÃO}

Ceratomileusis in situ associada à ceratectomia fotorrefrativa com excimer laser (LASIK) é um procedimento cirúrgico que se baseia na criação de um retalho corneal disciforme lamelar plano (flap), com o uso de microceratótomo, aplicação de excimer laser no leito estromal para ablação de tecido, seguida de reposicionamento do retalho corneal, dando novo contorno à superfície ocular, com finalidade de corrigir erros de refração ${ }^{(1-2)}$. O termo ceratomileusis para correção da miopia foi introduzido por José Barraquer, no início da década de $60^{(1)}$.

A obtenção de acuidade visual satisfatória pouco tempo após a cirurgia, o alto grau de satisfação do paciente, a correção de altos graus de miopia e o fato de não causar dano à camada de Bowman no centro da córnea contribuem para que seja atualmente o método de escolha entre os cirurgiões ${ }^{(3-7)}$. 
Apesar da sua eficácia e segurança, complicações relacionadas à confecção do retalho corneal podem ocorrer: retalho superficial e fino, incompleto, livre ou com buraco central, ceratite interlamelar difusa, crescimento epitelial na interface, astigmatismo irregular, hipercorreção e hipocorreção, infecção e depósitos na interface ${ }^{(2-3,8-13)}$.

O objetivo deste estudo foi comparar a superfície cortante de lâminas novas com lâminas reutilizadas no LASIK, pela microscopia eletrônica de varredura $(\mathrm{MEV})^{(14)}$, e estabelecer possíveis relações entre sua utilização seriada e eventual presença de substâncias orgânicas sobre a lâmina.

\section{MÉTODOS}

Foram obtidas e analisadas 25 lâminas cirúrgicas AccuGlide $^{\circledR}$ (Bausch Lomb, EUA), divididas em 3 grupos: Grupo 1 (controle), cinco lâminas novas e sem uso; Grupo 2, dez lâminas utilizadas duas vezes (olho direito seguido do olho esquerdo), em procedimento bilateral seriado; Grupo 3, dez lâminas utilizadas quatro vezes (quatro cirurgias em dois pacientes diferentes, considerando um procedimento bilateral seriado para cada paciente), lavadas com vapor de água e solução salina estéril e esterilizadas por óxido de etileno entre os procedimentos (ou seja, após o segundo uso da lâmina).

As lâminas reutilizadas dos Grupos 2 e 3 foram utilizadas e esterilizadas conforme o protocolo cirúrgico estabelecido pelo centro de cirurgia refrativa fornecedor das mesmas. As lâminas foram acondicionadas em recipientes apropriados fornecidos pelo fabricante e transportadas para o laboratório de MEV, onde foram submetidas à técnica de preparo e fotografadas.

Após colagem em suporte metálico cilíndrico com cola de cianoacrilato, as lâminas foram submetidas ao processo de metalização com vapor de ouro, visando aumentar a conductibilidade da superfície da amostra através da fina camada do metal. Em seguida, o ouro era removido por eletrodo maciço (cátodo), por bombardeamento com íons pesados de argônio no aparelho Blazers SCD 050, formando depósitos sobre as proeminências e reentrâncias da superfície da amostra.

Concluído o preparo, o material foi observado no microscópio eletrônico de varredura marca JEOL, modelo JSM 5300 (JEOL, Japão), sob alto vácuo e tensão aceleradora de elétrons com potência de $25 \mathrm{kV}$.

As lâminas eram mascaradas para o observador da MEV, que não sabia quantas vezes estas haviam sido utilizadas.

Cada lâmina foi observada em quatro pontos aleatórios, dois centrais e dois periféricos, com aumentos de 50, 350 e 1.000 vezes. Foram analisados os seguintes aspectos: presença de substâncias orgânicas, denteamento da superfície cortante, regularidade da superfície do corpo da lâmina e presença de material metálico sobre a lâmina. Foram feitas comparações, por análise descritiva, entre os grupos referentes aos pontos centrais e periféricos.

Todas as observações foram documentadas com máquina fotográfica acoplada ao próprio microscópio. Os resultados das observações mostram a presença ou ausência dos aspectos analisados e não a quantidade observada.

O método estatístico utilizado foi o teste Exato de Fisher para a comparação entre os Grupos 2 e 3 quanto a irregularidade e presença de substâncias orgânicas nos pontos centrais e periféricos e o de Wilcoxon, para amostras pequenas, na avaliação da análise descritiva entre os grupos. Foi considerado estatisticamente significante um valor descritivo menor do que 0,1 .

\section{RESULTADOS}

As lâminas sem uso (Grupo 1) apresentaram regularidade da superfície, bem como ausência de denteamento da superfície cortante, de substâncias orgânicas, e de material metálico em 20 dos 20 pontos observados (Figura 1). Nas lâminas reutilizadas (Grupos 2 e 3), não foram observados presença de material metálico ou denteamento da superfície cortante em nenhum ponto observado.

Entretanto, foram observadas irregularidades da superfície do corpo das lâminas reutilizadas (Figura 1). A tabela 1 demonstra que nas utilizadas duas vezes (Grupo 2), nove dos vinte pontos centrais (45\%) e doze dos vinte pontos periféri$\cos (60 \%)$ apresentaram irregularidades. Nas lâminas utilizadas quatro vezes (Grupo 3), este achado foi observado em dezessete dos vinte pontos centrais $(85 \%)$ e em dezenove dos vinte pontos periféricos $(95 \%)$ revelando que as lâminas do Grupo 3 apresentaram mais irregularidades do que as do Grupo 2 nos pontos centrais ( $p=0,0094)$ e periféricos $(p=0,0098)$. A análise estatística evidenciou ainda diferença significante entre o uso de duas (Grupo 2) ou quatro vezes (Grupo 3) nos pontos central 1 (nível descritivo 0,033) e periférico 1 (nível descritivo 0,087), com p<0,1. Porém, não foi observada diferença estatisticamente significante quando comparados os pontos central 2 e periférico 2 entre os Grupos 2 e 3 ( $p=0,370$ e $\mathrm{p}=0,303$, respectivamente) (Tabela 2).

Depósito de substância orgânica foi observado em todas as lâminas reutilizadas (Figura 1). A tabela 3 demonstra que naquelas utilizadas duas vezes (Grupo 2), dez dos vinte pontos centrais $(50 \%)$ e quinze dos vinte pontos periféricos $(75 \%)$ evidenciaram deposição deste tipo de material. O mesmo também foi encontrado nas lâminas utilizadas quatro vezes (Grupo 3), em dezessete dos vinte pontos centrais analisados (85\%), bem como em dezenove dos vinte pontos periféricos (95\%) revelando que as lâminas do grupo 3 apresentaram mais depósitos de substância orgânica do que as do grupo 2 nos pontos centrais ( $\mathrm{p}=0,0204)$ e periféricos $(\mathrm{p}=0,0909)$. A análise estatística não mostrou diferença significante entre o uso de duas ou quatro vezes em relação aos pontos central 2 e periféricos 1 e 2 , mas houve diferença estatisticamente significante em relação ao ponto central 1 (nível descritivo 0,087) (Tabela 4).

Os Grupos 2 e 3 apresentaram diferenças estatisticamente significantes quanto à irregularidade da superfície e à presença 
de substâncias orgânicas quando comparados ao Grupo 1 (lâminas sem uso) nos pontos central e periférico 1 e 2 ( $\mathrm{p}<0,001)$.

\section{DISCUSSÃO}

O presente estudo não demonstrou alterações visíveis do material metálico após reutilização da lâmina do microceratótomo até quatro vezes. Por outro lado, evidenciamos a presença de restos orgânicos nas lâminas reutilizadas, o que poderia representar possível fator etiológico de complicações pósoperatórias como inflamação e ceratite lamelar difusa (DLK).

DLK é uma condição não infecciosa que pode ser observada na interface após $\operatorname{LASIK}^{(9-10,15-17)}$. Classicamente ocorre alguns dias após o procedimento em casos isolados ou em clusters epidemiológicos e está relacionada com possíveis agentes ou eventos, como debris na interface, sangue, óleo do microceratótomo, iodopovidona, carboximetilcelulose, soluções de limpeza e endotoxinas bacterianas ${ }^{(18-19)}$.

Inclusões na interface após LASIK são descritos na literatura $^{(9-10,19-20)}$. Behrens et al estudando experimentalmente a degradação da qualidade do corte de lâminas cirúrgicas após uso repetido (até cinco vezes), notaram progressiva perda de regularidade e acúmulo de material orgânico e cristais de solução salina $^{(21)}$. Com quatro vezes de uso, notaram corrosão da superfície cortante com irregularidades, denteamento e dobras.

É de se destacar que, em nosso estudo, mesmo após utilização de métodos de limpeza como o vapor e irrigação com solução salina estéril e esterilização com óxido de etileno, não houve eliminação total de resíduos orgânicos. No presente estudo, observou-se que mesmo com os procedimentos de limpeza efetuados após o segundo uso entre dois pacientes do Grupo 3, houve maior número de pontos com irregularidades e depósitos de substância orgânica do que no Grupo 2 eviden-
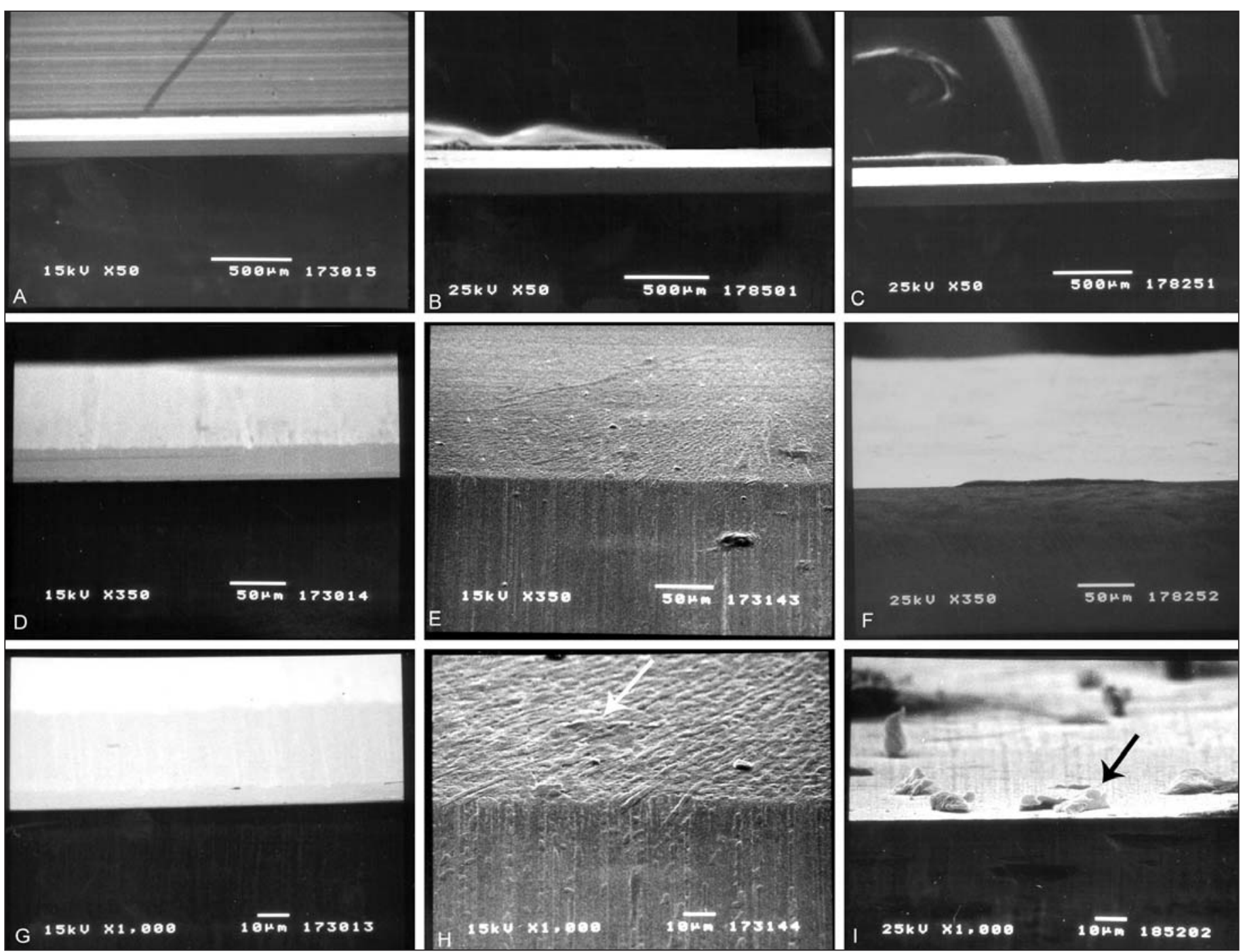

G $15 \mathrm{kU} \times 1$,

$1 \overline{\mu_{m}} \quad 173813$

Figura 1 - Microscopia eletrônica de varredura dos 3 grupos analisados. A, D e G: grupo 1 (lâmina sem uso). B, E e H: grupo 2 (lâminas usadas 2 vezes) evidencia irregularidade do corpo da lâmina e presença de substância orgânica (seta). C, F e l: grupo 3 (lâminas usadas 4 vezes) apresenta achados semelhantes àqueles encontrados no grupo 2. Aumentos utilizados para documentação fotográfica: $50 x(A, B, C), 350 x(D, E, F)$ e $1000 x(G, H, I)$. 
ciando a não eficácia deste tipo de conduta (Tabelas 1 e 3). Este material remanescente pode depositar-se sobre a superfície estromal da interface do retalho corneal, promovendo as mais variadas e imprevisíveis inconveniências, como deposição de células epiteliais e endotoxinas.

Apesar de descartáveis, lâminas cirúrgicas são freqüentemente utilizadas em cirurgias bilaterais e simultâneas, podendo estar relacionadas à gênese de complicações. De acordo com o primeiro censo brasileiro em cirurgia refrativa, $6 \%$ dos entrevistados trocam a lâmina do microceratótomo entre diferentes olhos, $32,5 \%$ referem trocar entre pacientes, $40,7 \%$ trocam-na entre 2 e 3 pacientes, $15,8 \%$ entre 4 e 6 pacientes, $2,9 \%$ entre 7 e 10 pacientes, $0,9 \%$ em mais de 10 pacientes, e 1,2\% apenas trocam-na diariamente ${ }^{(22)}$.

$\mathrm{Na}$ literatura fornecida pelos fabricantes das lâminas, há orientação explícita para uso único e posterior descarte. Embora o grau de deterioração e qualidade de corte não pareça

$\begin{aligned} & \text { Tabela 1. Irregularidade da superfície das lâminas cirúrgicas dos } \\
& \text { grupos } 2 \text { e } \mathbf{3} \text { observadas pela MEV }\end{aligned}$
\begin{tabular}{lcc} 
Pontos centrais & Pontos periféricos \\
Uso & $\mathbf{N}(\%)$ & $\mathbf{N}(\%)$ \\
$2 \mathrm{X}$ & $9(45)$ & $12(60)$ \\
$4 \mathrm{X}$ & $17(85)$ & $19(95)$ \\
valor do $p^{*}$ & 0,0094 & 0,0098 \\
${ }^{*}$ Teste Exato de Fischer & \\
\hline
\end{tabular}

\begin{tabular}{|c|c|c|}
\hline Ponto & Pontos centrais & Pontos periféricos \\
\hline 1 & $0,033^{*}$ & $0,087^{*}$ \\
\hline 2 & 0,370 & 0,303 \\
\hline${ }^{*} p<0,1$ & & \\
\hline
\end{tabular}

\begin{tabular}{|c|c|c|}
\hline Uso & $\begin{array}{c}\text { Pontos centrais } \\
\text { N (\%) }\end{array}$ & $\begin{array}{c}\text { Pontos periféricos } \\
\mathrm{N}(\%)\end{array}$ \\
\hline $2 x$ & $10(50)$ & $15(75)$ \\
\hline $4 X$ & 17 (85) & 19 (95) \\
\hline valor do $p^{*}$ & 0,0204 & 0,0909 \\
\hline
\end{tabular}

\begin{tabular}{|c|c|c|}
\hline Ponto & Pontos centrais & Pontos periféricos \\
\hline 1 & $0,087^{*}$ & 0,474 \\
\hline 2 & 0,370 & 0,582 \\
\hline
\end{tabular}

ser influenciado após quatro utilizações da mesma lâmina, acreditamos que a presença de tecido orgânico remanescente sobre a superfície da lâmina limite sua reutilização.

$\mathrm{O}$ alto custo financeiro da preparação do material e a dificuldade em analisar o material na MEV foram os fatores responsáveis pelo tamanho reduzido da amostra utilizado neste estudo.

\section{CONCLUSÃO}

Nossos resultados demonstram depósito de material orgânico e irregularidades na superfície das lâminas reutilizadas. Por outro lado, não observamos alterações da borda cortante do material metálico após reutilização da lâmina do microceratótomo até quatro vezes. As implicações clínicas destes achados precisam ser avaliadas devido à possibilidade de estarem relacionados com complicações pós-operatórias interlamelares comumente encontradas no LASIK.

\section{ABSTRACT}

Purpose: To evaluate and compare, by scanning electron microscopy (SEM), the sharpness of the edge of brand new surgical blades and consecutively used surgical blades in laserassisted in situ keratomileusis (LASIK). Methods: Tewnty-five AccuGlide $^{\circledR}$ (Bausch \& Lomb, USA) surgical blades were evaluated, divided in to 3 groups: Group 1 (control), non-used surgical blades; Group 2, ten surgical blades used twice (simultaneous bilateral procedure); Group 3, ten surgical blades used four times (two consecutive simultaneous bilateral procedures). Each blade was analyzed by SEM at four random points, two central and two peripheral points, with magnification of 50x, 350x, and 1,000x. Results: Blade body irregularities and organic material were observed on all used surgical blades (Groups 2 and 3), but not on new surgical blades (Group 1). In addition, Group 3 revealed more irregularities in both central $(\mathrm{p}=0.0094)$ and peripheral points $(\mathrm{p}=0.0098)$ than Group 2, as well as organic material deposition $(\mathrm{p}=0.0204$ and $\mathrm{p}=0.0909$, respectively). Neither metalic material nor irregularities in the cutting edge of the blade were observed in any group. Conclusion: No difference regarding the cutting edge was observed between new and blades reused up to four times, however irregularities and organic material were observed in all reused blades. Reusing blades for LASIK may produce structural changes and its clinical implications need to be evaluated due to the possible relation with interlamellar postoperative complications.

Keywords: Keratomileusis, laser in situ; Laser surgery; Postoperative complications; Inflammation

\section{REFERÊNCIAS}

1. Barraquer JI. Queratomileusis para la corrección de la miopia. Arch Soc Am Oftal Optom. 1964;5:27-48. 
2. Filatov V, Vidaurri-Leal JS, Talamo JH. Selected complications of radial keratotomy, photorefractive keratectomy, and laser in situ keratomileusis. In Ophthalmol Clin. 1997;37(1):123-48. Review.

3. Gimbel HV, Penno EE, van Westenbrugge JA, Ferensowicz M, Furlong MT Incidence and management of intraoperative and early postoperative complications in 1000 consecutive laser in situ keratomileusis cases. Ophthalmology. 1998;105(10):1839-47; discussion 1847-8.

4. Jones SS, Azar RG, Cristol SM, Geroski DH, Waring GO $3^{\text {rd }}$, Stulting RD, et al. Effects of laser in situ keratomileusis (LASIK) on the corneal endothelium. Am J Ophthalmol. 1998;125(4):465-71.

5. Leaming DV. Practice styles and preferences of ASCRS members - 2003 survey. J Cataract Refract Surg. 2004;30(4):892-900.

6. Solomon KD, Fernandez de Castro LE, Sandoval HP, Bartholomew LR, Vroman DT. Refractive surgery survey 2003. J Cataract Refract Surg. 2004;30(7):1556-69.

7. Sugar A, Rapuano CJ, Culbertson WW, Huang D, Varley GA, Agapitos PJ, et al. Laser in situ keratomileusis for myopia and astigmatism: safety and efficacy: a report by the American Academy of Ophthalmology. Ophthalmology. 2002;109(1):175-87.

8. Wilson SE. LASIK: management of common complications. Laser in situ keratomileusis. Cornea. 1998;17(5):459-67.

9. Casanova FH. Ceratite lamelar difusa pós-LASIK. In: Alves MR, Chamon W, Nosé W. Cirurgia refrativa. Rio de Janeiro: Cultura Médica; 2003. p.431-7.

10. Smith RJ, Malloney RK. Diffuse lamellar keratitis. A new syndrome in lamellar refractive surgery. Ophthalmology. 1998;105(9):1721-6.

11. Helena MC, Meisler D, Wilson SE. Epithelial growth within the lamellar interface after laser in situ keratomileusis (LASIK). Cornea. 1997;16(3):300-5.

12. Stulting RD, Carr JD, Thompson KP, Waring GO $3^{\text {rd }}$, Wiley WM, Walker
JG. Complications of laser in situ keratomileusis for the correction of myopia. Ophthalmology. 1999;106(1):13-20.

13. Hirst LW, Vandeleur KW Jr. Laser in situ keratomileusis interface deposits. J Refract Surg. 1998;14(6):653-4.

14. Haddad A. Técnicas de microscopia eletrônica de varredura. Rio de Janeiro: Sociedade Brasileira de Microscopia Eletronica, 1998. p.33.

15. Holland SP. Update in cornea and external disease: solving the mystery of "sands of the Sahara" syndrome (diffuse lamellar keratitis). Can J Ophthalmol. 1999;34(4):193-4.

16. Holland SP, Mathias RG, Morck DW, Chiu J, Slade SG. Diffuse lamellar keratitis related to endotoxins released from sterilizer reservoir biofilms. Ophthalmology. 2000;107(7):1227-33; discussion 1233-4.

17. Kaufman SC, Maitchouk DY, Chiou AG, Beuerman RW. Interface inflammation after laser in situ keratomileusis. Sands of the Sahara syndrome. J Cataract Refract Surg. 1998;24(12):1589-93.

18. Harrison DA, Periman LM. Diffuse lamellar keratitis associated with recurrent corneal erosions after laser in situ keratomileusis. J Refract Surg. 2001;17(4):463-5.

19. Hoffman RS, Fine IH, Packer M, Reynolds TP, Bebber CV. Surgical gloveassociated diffuse lamellar keratitis. Cornea. 2005;24(6):699-704.

20. Vinciguerra P, Azzolini M, Radice P, Sborgia M, De Molfeta V. A method for examining surface and interface irregularities after photorefractive keratectomy and laser in situ keratomileusis: predictor of optical and functional outcomes. J Refract Surg. 1998;14(2 Suppl):S204-6.

21. Behrens A, Seitz B, Langenbucher A, Kus MM, Rummelt C, Kuchle M. Evaluation of corneal flap dimensions and cut quality using a manually guided microkeratome. J Refract Surg. 1999;15(2):118-23.

22. Victor G, Urbano A, Marçal S, Porto R, Francesconi CM, Forseto AS, et al. Primeiro censo brasileiro em cirurgia refrativa. Arq Bras Oftalmol. 2005;68 (6):727-33 